\title{
Vitamin B12 Deficiency Presenting With Microangiopathic Hemolytic Anemia
}

\author{
Haitham Osman ${ }^{1}$, Turki A. Alwasaidi ${ }^{1,2}$, Abdulqader Al-Hebshi ${ }^{3,4}$, Najah Almutairi ${ }^{1}$, Hussein Eltabbakh \\ 5
}

1. Hematology, Prince Mohammed Bin Abdulaziz Hospital/Ministry of National Guard Health Affairs, Madinah, SAU 2. Internal Medicine, Taibah University, Madinah, SAU 3. Pediatric Hematology Oncology, Prince Mohammed Bin Abdulaziz Hospital/Ministry of National Guard Health Affairs, Madinah, SAU 4. Pediatrics, King Saud Bin Abdulaziz University for Health Sciences, Riyadh, SAU 5. Internal Medicine, Prince Mohammed Bin Abdulaziz Hospital/Ministry of National Guard Health Affairs, Madinah, SAU

Corresponding author: Haitham Osman, haithmm@gmail.com

\begin{abstract}
Vitamin B12 has essential roles in DNA synthesis, red blood cell development, and neurologic functions. Vitamin B12 deficiency is relatively common, particularly in people aged over 60 years. Among hematological disturbances, microangiopathic hemolytic anemia with thrombocytopenia or so-called pseudo-thrombotic microangiopathy (pseudo-TMA) is a particularly rare but significant clinical complication in patients with vitamin B12 deficiency. We herein describe a case of an elderly patient with pseudo-TMA whose lack of vitamin B12 was misdiagnosed as thrombotic thrombocytopenic purpura (TTP). The patient was admitted as a case of pancytopenia with a hemolytic picture. The initial impression was TTP versus acute promyelocytic leukemia M3. After examination of laboratory tests and bone marrow examination, we deduced that the patient had a B12 deficiency. The condition of the patient improved with B12 replacement. This report should remind physicians to widen their differential diagnoses when patients present with microangiopathic hemolysis or in patients who are not responsive to standard treatments for TTP.
\end{abstract}

Review began $12 / 08 / 2020$ Review ended 12/26/2020 Published 01/10/2021

๑) Copyright 2021 Osman et al. This is an open access article distributed under the terms of the Creative Commons Attribution License CC-BY 4.0., which permits unrestricted use, distribution, and reproduction in any medium, provided the original author and source are credited.
Categories: Internal Medicine, Oncology, Hematology

Keywords: schistocytes, microangiopathic hemolytic anemia, thrombotic thrombocytopenic purpura, pseudo-tma, vitamin b12 deficiency

\section{Introduction}

Every vitamin is assigned a specific and unique role in the human body, for instance, "vitamin B12" is one of the most vital vitamins with its unique structure and composition of the mineral cobalt and thus the origin of the name cobalamin. It possesses various roles on different levels, including DNA and red blood cell (RBC) synthesis, in addition to several neurologic functions [1]. The cut-off was defined by the World Health Organization, where cobalamin deficiency was presented as less than $150 \mathrm{pmol} / \mathrm{L}[2]$. During the recent decade, vitamin B12 deficiency has become relatively common, particularly in the population aged over 60 years [3]. A broad range of vitamin B12 deficiency-related clinical evidence has been reported describing clinical severity ladder, ranging from fatigue, anemia, glossitis, and subtle neurologic disturbance in mildto-moderate cases, to severe hematological abnormalities, severe neurologic manifestations, and/or cardiomyopathy in severe cases [3]. Microangiopathic hemolytic anemia (MAHA) with thrombocytopenia or so-called pseudo-thrombotic microangiopathy (pseudo-TMA) is a particularly significant hematological complication in patients with cobalamin deficiency $[4,5]$. Damaged RBC membrane can cause intravascular hemolysis, leading to MAHA, as characterized by the appearance of schistocytes (key characteristics of MAHA) [6]. Primary thrombotic microangiopathy syndromes involve serious conditions such as thrombotic thrombocytopenic purpura (TTP), hemolytic uremic syndrome, drug-induced TMA, and complementmediated TMA. These conditions must be managed and controlled immediately, therefore uncovering the primary etiology including plasmapheresis or monoclonal antibodies that bind complement proteins $[7,8]$. One of the cobalamin deficiency-TMA features is that patients do not respond to plasma infusion or exchange; the failure to recognize this diagnosis may prompt unnecessary treatments [2]. TTP is a quickly advancing and life-threatening illness that, in past years, featured a classic pentad of MAHA, thrombocytopenia, fever, renal dysfunction, and neurologic abnormalities [9]. Patients with malignancies, as well as those with autoimmune disorders and following solid organ and stem cell transplants, may present with thrombocytopenia and MAHA. In this case, the treatment should be directed at the specific underlying condition [6]. Cobalamin deficiency-induced TMA designates TMA secondary to vitamin B12 deficiency. Usually, cases with pseudo-TMA present with hemolytic anemia, thrombocytopenia, and dysmorphic "fragmented" RBCs. They are often misdiagnosed to have other TMA syndromes and receive unnecessary therapy such as plasmapheresis $[1,2]$. We herein describe a case of an elderly patient with pseudo-TMA whose lack of vitamin B12 was misdiagnosed with TTP.

\section{Case Presentation}




\section{Cureus}

An 84-year-old married male presented to the outpatient clinic for a routine annual check-up. The patient had hypertension and hypothyroidism. He had no change in his bowel habits, no melena or bleeding from any site, no weight loss, no loss of appetite, no fever, no shortness of breath, no headache or other neurologic symptoms, no chest pain, palpitation, or other cardiovascular complaints, and no urinary symptoms. He underwent bowel resection due to intestinal obstruction. Accordingly, he was taking amlodipine $5 \mathrm{mg}$, thyroxine $75 \mathrm{mcg}$, and aspirin $81 \mathrm{mg}$ daily. He had no history of smoking, alcohol, or drug use, nor a family history of hematological disease. On physical examination, the patient was fully conscious oriented; his body temperature was $36.8^{\circ} \mathrm{C}$, blood pressure was $131 / 59 \mathrm{mmHg}$, pulse was 78 beats per minute, respiratory rate was 18 breaths per minute, and oxygen saturation was $98 \%$ on room air. He was conscious, oriented, and slightly pale, and had no jaundice or nail changes. His head and neck examination showed no oral ulcers, a normal tongue, no lymphadenopathy, and no peripheral stigmata of chronic liver disease; his jugular venous pressure was not raised, and cardiovascular and respiratory examinations were normal. There were midline abdominal longitudinal and right sub-costal scares; no organomegaly was present. There was a bilateral petechial rash on the anterior aspect of both legs, extending from the knee joint to the ankle joint. His neurologic and musculoskeletal examinations were normal.

His initial laboratory finding (Table 1 ) revealed pancytopenia with a hemoglobin of $8.2 \mathrm{~g} / \mathrm{dL}$ (normal: 11.0$14.5 \mathrm{~g} / \mathrm{dL}$ ), platelets of $64 \times 10^{9} / \mathrm{L}$ (normal: $150-450 \times 10^{9} / \mathrm{L}$ ), WBCs of $1.6 \times 10^{9} / \mathrm{L}$ (normal: $4-12 \times 10^{9} / \mathrm{L}$ ), absolute neutrophilic count of $0.35 \times 10^{9} / \mathrm{L}$ (normal: $2-7.5 \times 10^{9} / \mathrm{L}$ ), and absolute lymphocytic count of $0.98 \times$ 109 / (normal: $\left.1.0-4.4 \times 10^{9} / \mathrm{L}\right)$.

\begin{tabular}{|c|c|c|c|}
\hline Parameters & Reference values & Results upon admission & Results after one month of treatment \\
\hline Hgb & $11.0-14.5 \mathrm{~g} / \mathrm{dL}$ & 8.2 & 10 \\
\hline Hct & $0.31-0.45 \mathrm{~L} / \mathrm{L}$ & 0.253 & 0.319 \\
\hline RBC & $3.9-5.6 \times 10^{12} / \mathrm{L}$ & 2.74 & 3.28 \\
\hline MCV & $75-89 \mathrm{fL}$ & 114 & 97.1 \\
\hline $\mathrm{MCH}$ & $25-30 \mathrm{pg}$ & 35 & 30.7 \\
\hline Platelet & $150-450 \times 10^{\%} / \mathrm{L}$ & 64 & 273 \\
\hline WBC & $4-12 \times 10^{9 / L}$ & 1.6 & 8 \\
\hline ANC & $2-7.5 \times 10^{9} / \mathrm{L}$ & 0.35 & 4.46 \\
\hline ALC & $1-4.4 \times 10^{9} / \mathrm{L}$ & 0.98 & 2.11 \\
\hline D-Dimer & $0.00-0.5 \mathrm{mg} / \mathrm{mL}$ & 17.44 & 2.49 \\
\hline Schistocytes & Less than $0.2 \%$ & $5 \%$ & Less than $1 \%$ \\
\hline Reticulocyte & $0.5-1.5 \%$ & 2.22 & 5.61 \\
\hline Fibrinogen & $1.5-4.1 \mathrm{pm} / \mathrm{L}$ & 1.11 & 2.5 \\
\hline LDH & $125-220 \mathrm{U} / \mathrm{L}$ & 486 & 365 \\
\hline Vitamin B12 & 138-652 pmo//L & 61 & 530 \\
\hline
\end{tabular}

\section{TABLE 1: Laboratory Investigations}

$\mathrm{Hgb}$, hemoglobin; Hct, hematocrit; RBC, red blood cells; MCV, mean corpuscular volume; $\mathrm{MCH}$, mean corpuscular hemoglobin; MCV, mean corpuscular volume; WBC, white blood cells; ANC, absolute neutrophilic count; ALC, absolute lymphocytic count; LDH, lactate dehydrogenase

The peripheral blood smear showed macrocytosis, with frequent schistocytes of $5 \%$, and hyper-segmented neutrophils (Figure 1). The lactate dehydrogenase (LDH) was $486 \mathrm{U} / \mathrm{L}$ (high; normal: 125-220 U/L), total bilirubin was 45 umol/L (high; normal: 0-18 umol/L), haptoglobin was <0.058 g/L (low; normal: 0.36-1.95 $\mathrm{g} / \mathrm{L}$ ), reticulocytes count was $2.2 \%$ (normal: $0.5-1.5 \%$ ); B12, folate levels, viral serology, and autoimmune workup was also conducted. The patient underwent bone marrow aspiration and trephine biopsy, and there was no evidence of acute myeloid leukemia or infiltrative bone marrow. 


\section{Cureus}

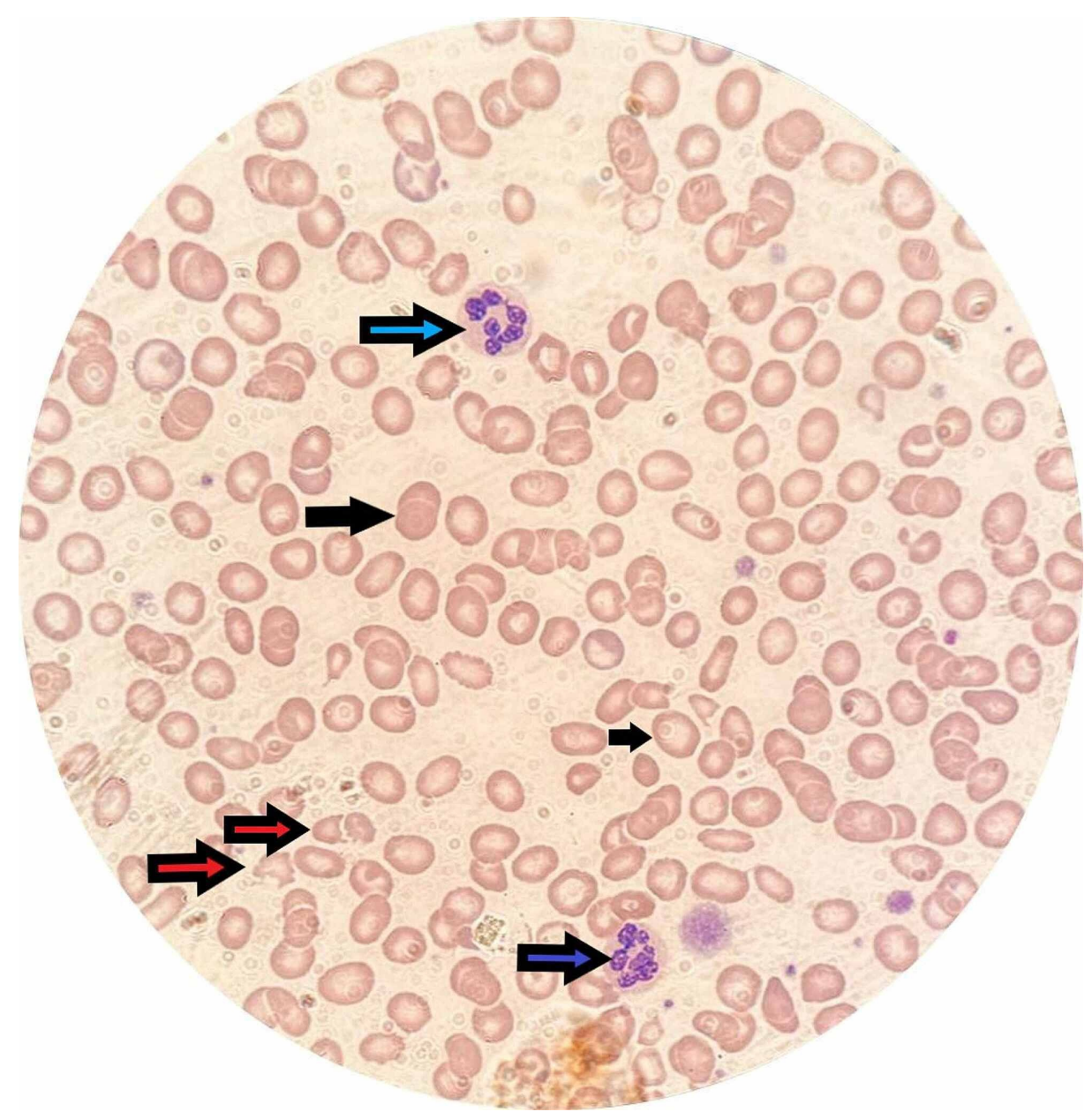

FIGURE 1: Peripheral blood smear showing schistocytes (red arrows), hypersegmented neutrophils (blue arrows), and macrocytes (black arrows)

As per the available laboratory results, the patient was initially diagnosed with MAHA. Though the full pentad of TTP was not fully matched and rest of the laboratory workup results were awaited, he was started empirically on fresh frozen plasma (FFP) transfusion every six hours while ADAMTS13 (a disintegrin and metalloproteinase with a thrombospondin type 1 motif, member 13) was sent for workup. However, after 72 hours of FFP, his blood count did not improve and hemolytic markers were rising. Pan CT was performed showing no hidden malignancy that could trigger MAHA. On the third day of admission, the vitamin B12 level result came back with $61 \mathrm{pmol} / \mathrm{L}$ (normal: 138-652 pmol/L); his folate level was normal. He has a normal level of ADAMTS13, normal homocysteine level, and negative anti-intrinsic and anti-parietal cell antibodies.

Finally, we deduced that the patient has severe vitamin B12 deficiency; therefore he was started on intramuscular cyanocobalamin $1,000 \mathrm{mcg}$ once daily for seven days and then changed to once weekly for five weeks and then monthly lifelong.

The patient showed clinical improvement at day 3 of parenteral replacement of vitamin B12; his white blood cells increased to $3.5 \times 10^{9} / \mathrm{L}$, hemoglobin to $10 \mathrm{~g} / \mathrm{dL}$, and platelet to $100 \times 10^{9} / \mathrm{L}$. The schistocytes started to disappear gradually. The complete blood count and vitamin B12 levels were normalized after one month of treatment (Table 1).

\section{Discussion}

This is a detailed case of pseudo-TMA due to extreme vitamin B12 deficiency following bowel resection (terminal ileum). Previous evidence showed that the maintenance of the terminal ileum may protect vitamin B12 retention capacity [10]. Moreover, vitamin B12 deficiency-induced TMA postures a real challenge for professionals managing cases of thrombocytopenia, hemolytic anemia, and schistocytosis. In spite of the fact that the differential diagnosis should aim at ruling out the foremost critical conditions in the first place, estimation of vitamin B12 and methylmalonic level to the current symptomatic board for the assessment of 
TTP can guide clinicians to appropriate determination and treatment [11]. As mentioned, vitamin B12 plays a significant role in RBC synthesis; therefore, when this compound reaches low levels (cobalamin deficiency), the rigidity of RBC membrane increases and the erythrocyte deformability decreases, causing the lysis of RBCs [12]. Furthermore, cobalamin deficiency not only affects RBCs but also pauses the maturation of all cell lines in the marrow. It can manifest with hemolytic anemia secondary to abnormal erythropoiesis and indirect hyperbilirubinemia, yet it does not often manifest with MAHA. Past literature contains only very few cases of vitamin B12 deficiency-induced MAHA, termed as "pseudo-thrombotic angiopathy." Others proposed that severe hyperhomocysteinemia combined with vitamin B12 deficiency points to an impressive peripheral blood smear and clinical findings similar to TTP [13].

TTP is the most differential diagnosis of pseudo-TMA that causes a hurdle for the professionals. Although TTP can be deadly without any proper therapy plan, starting plasmapheresis treatment for TTP and vitamin B12 replacement for cobalamin deficiency might be a plausible choice for pseudo-TMA-suspected cases [14]. One of the features that assist in primary diagnosis is that pseudo-TMA does not respond to FFP, which was the case in our report [1].

Vitamin B12 deficiency-related hemolytic anemia may cause hyperbilirubinemia (due to the destruction of RBCs that have not achieved maturation in the marrow), or may also cause extravascular hemolysis, which should not result in microangiopathy [13]. Moreover, the patient in this report presented with frequent appearance of schistocytes on the peripheral blood smear, in addition to elevated bilirubin and LDH levels.

Furthermore, most of our case's clinical findings aligned with the standard manifestations and laboratory findings of pseudo-TMA previously described by Andrès et al., including hemolytic anemia, hemoglobin (5.1 to $10 \mathrm{~g} / \mathrm{dL})$, mean corpuscular volume (112 to $124 \mathrm{fL})$, platelet count $(25,000$ to $11,0000 / \mu \mathrm{L})$ and vitamin B12 level (12 to $70 \mathrm{pg} / \mathrm{mL})[15]$.

\section{Conclusions}

TMA due to vitamin B12 deficiency is a rare condition, yet it must be inspected in all patients with clinical and laboratory manifestations of TTP. Our case highlights the importance of differential diagnoses of vitamin B12 deficiency in cases presenting microangiopathic hemolysis or in cases that are not responsive to standard treatments for TTP.

\section{Additional Information \\ Disclosures}

Human subjects: Consent was obtained by all participants in this study. Conflicts of interest: In compliance with the ICMJE uniform disclosure form, all authors declare the following: Payment/services info: All authors have declared that no financial support was received from any organization for the submitted work. Financial relationships: All authors have declared that they have no financial relationships at present or within the previous three years with any organizations that might have an interest in the submitted work. Other relationships: All authors have declared that there are no other relationships or activities that could appear to have influenced the submitted work.

\section{References}

1. Fahmawi Y, Campos Y, Khushman M, et al.: Vitamin B12 deficiency presenting as pseudo-thrombotic microangiopathy: a case report and literature review. Clin Pharmacol Adv Appl. 2019, 11:127-131. 10.2147/CPAA.S207258

2. Tran PN, Tran MH: Cobalamin deficiency presenting with thrombotic microangiopathy (TMA) features: a systematic review. Transfus Apher Sci. 2018, 57:102-106. 10.1016/j.transci.2018.01.003

3. Hunt A, Harrington D, Robinson S: Vitamin B12 deficiency. BMI. 2014, 349:5226. 10.1136/bmj.g5226

4. Noël N, Maigné G, Tertian G, et al.: Hemolysis and schistocytosis in the emergency department: consider pseudothrombotic microangiopathy related to vitamin B12 deficiency. QJM Mon J Assoc Physicians. 2013, 106:1017-1022. 10.1093/qjmed/hct142

5. Harada Y, Komori I, Morinaga K, Shimizu T: Microangiopathic haemolytic anaemia with thrombocytopenia induced by vitamin B12 deficiency long term after gastrectomy. BMJ Case Rep. 2018, 2018:225915. 10.1136/bcr-2018-225915

6. Jagadish Kumar K, Chowdary D, Shetty AU, Anitha C: Microangiopathic hemolytic anemia - a rare presentation of vitamin B12 deficiency. Indian J Pediatr. 2018, 85:322-324. 10.1007/s12098-017-2491-1

7. George JN, Nester CM: Syndromes of thrombotic microangiopathy. N Engl J Med. 2014, 371:654-666. 10.1056/NEJMra1312353

8. Scully M, Cataland S, Coppo P, et al.: Consensus on the standardization of terminology in thrombotic thrombocytopenic purpura and related thrombotic microangiopathies. J Thromb Haemost. 2017, 15:312322. 10.1111/jth.13571

9. Yousaf F, Spinowitz B, Charytan C, Galler M: Pernicious anemia associated cobalamin deficiency and thrombotic microangiopathy: case report and review of the literature. Case Rep Med. 2017, 2017:9410727. $10.1155 / 2017 / 9410727$

10. Thompson WG, Wrathell E: The relation between ileal resection and vitamin B12 absorption . Can J Surg. 1977, 20:461-464. 


\section{Cureus}

11. Schwartz J, Winters JL, Padmanabhan A, et al.: Guidelines on the use of therapeutic apheresis in clinical practice-evidence-based approach from the Writing Committee of the American Society for Apheresis: the sixth special issue. J Clin Apheresis. 2013, 28:145-284. 10.1002/jca.21276

12. Tuten N, Bennett C, Babcock W: Thrombotic thrombocytopenic purpura or cobalamin deficiency? A case report and review. Clin Case Rep Rev. 2015, 2015:1. 10.15761/CCRR.1000152

13. Panchabhai TS, Patil PD, Riley EC, Mitchell CK: When the picture is fragmented: vitamin B12 deficiency masquerading as thrombotic thrombocytopenic purpura. Int J Crit Illn Inj Sci. 2016, 6:89-92. 10.4103/2229 5151.183026

14. Walter K, Vaughn J, Martin D: Therapeutic dilemma in the management of a patient with the clinical picture of TTP and severe B12 deficiency. BMC Hematol. 2015, 15:16. 10.1186/s12878-015-0036-2

15. Andrès E, Affenberger S, Zimmer J, et al.: Current hematological findings in cobalamin deficiency. A study of 201 consecutive patients with documented cobalamin deficiency. Clin Lab Haematol. 2006, 28:50-56. 10.1111/j.1365-2257.2006.00755.x 